\title{
Clima urbano e poluição sonora: revisão da literatura a partir da abordagem integrada
}

Urban climate and noise pollution: a review of the literature from the integrated approach

Article Info:

Article history: Received 2021-03-29 / Accepted 2021-03-29 / Available online 2021-03-30

doi: 10.18540/jcecvl7iss1pp11988-01-12e

Janaína Costa Lima

ORCID: https://orcid.org/0000-0003-3874-9394

Universidade Federal de Alagoas, UFAL, Brasil

E-mail: janacostalima@ hotmail.com

Jordana Teixeira da Silva

ORCID: https://orcid.org/0000-0002-7158-4766

Instituto Federal de Alagoas, IFAL, Brasil

E-mail: jordana.teixeira@ifal.edu.br

Stella Rosane da Silva Oliveira

ORCID: https://orcid.org/0000-0001-7627-6650

Universidade Federal de Alagoas, UFAL, Brasil

E-mail: stellarosane@ gmail.com

Ricardo Victor Rodrigues Barbosa

ORCID: https://orcid.org/0000-0003-4971-6037

Universidade Federal de Alagoas, UFAL, Brasil

E-mail: rvictor@fau.ufal.br

Maria Lucia Gondim da Rosa Oiticica

ORCID: https://orcid.org/0000-0001-9393-5427

Universidade Federal de Alagoas, UFAL, Brasil

E-mail: mloiticica@hotmail.com

\section{Resumo}

O crescimento urbano desordenado compromete a qualidade ambiental, incluindo os campos térmico e acústico. Esta pesquisa traz um panorama da produção científica sobre clima urbano e poluição sonora, que são frequentemente estudados isoladamente. Através de buscas em bases de dados científicos, seleção e análise dos artigos, observou-se escassez de trabalhos com abordagem integrada. Destaca-se a necessidade de trabalhos que relacionem os inúmeros aspectos ambientais, visando cidades saudáveis.

Palavras-chave: Revisão bibliográfica. Climatologia urbana. Acústica Ambiental.

\begin{abstract}
Urban expansion without planning compromises environmental quality, including thermal and acoustic fields. This research provides an overview of the scientific production on urban climate and noise pollution, which are often studied in isolation. Through searches of scientific databases, selection and analysis of articles, there was a shortage of works with an integrated approach. The need for work that relates the numerous environmental aspects, aiming at healthy cities, is highlighted.
\end{abstract}

Keywords: Bibliographical review. Urban climatology. Environmental Acoustics. 


\section{Introdução}

Atualmente, mais da metade da população mundial vive em áreas urbanas e estima-se que, entre 2018 e 2050, haverá mais 2,5 bilhões de habitantes urbanos (UN, 2019). A urbanização é um processo em escala global de intervenção humana que modifica a paisagem social e ambiental em todos os continentes e é resultado da migração da população das áreas rurais e do crescimento demográfico urbano natural (WHO, 2020). A falta de planejamento estratégico e o crescimento rápido urbano causam impactos ambientais e podem degradar a qualidade de vida dos moradores, tornando-se uma questão de saúde pública.

A poluição atmosférica e sonora são, respectivamente, as principais causadoras de doenças nos centros urbanos devido ao crescimento populacional e aumento do tráfego de veículos, principal fonte de ruído urbano. Os efeitos da exposição à poluição no ar na saúde, em curto ou longo prazo, variam desde o aumento de internações hospitalares e visitas de emergência ao aumento do risco de morte prematura. Em crianças e adultos, a poluição do ar pode levar à redução da função pulmonar, infecções respiratórias, agravamento da asma e também afetam a diabetes e o desenvolvimento neurológico (WHO, 2018). Os efeitos do ruído causados no homem envolvem: perda auditiva, estresse, distúrbio do sono, aborrecimento, zumbido, doenças cardiovasculares, derrame cerebral, tendência à depressão, entre outros (WHO, 2011).

Além disso, cidades com alta densidade de edificações e concentração de rodovias, podem causar as denominadas ilhas de calor urbanas, fenômeno associado ao clima da cidade com alteração das condições microclimáticas: aumento da temperatura do ar e da superfície, redução da umidade relativa, alteração na velocidade dos ventos, entre outros. A intensidade da ilha de calor possui forte relação com a morfologia urbana (Romero et al., 2019), assim como a acústica urbana, uma vez que a densidade construtiva, os perfis de ruas, as áreas verdes e a espacialização e forma dos edifícios alteram a permeabilidade acústica (Guedes et al., 2011).

Guedes et al. (2011), em estudo realizado na cidade de Aracaju/SE (Brasil), analisaram uma área de edifícios residenciais localizados próximos às vias de fluxo intenso de veículos. Através de medições, simulações computacionais e predições acústicas, observou-se que em regiões mais densas, os edifícios se comportam como barreiras de ruído, gerando sombra acústica, área mais silenciosa, no centro da quadra.

Já na pesquisa elaborada por Prata et al. (2019), foi analisado o estresse bioclimático em cidade tropical densa, na qual a verticalização de um centro de serviços, ao norte da cidade de Fortaleza/CE (Brasil) reduziu a velocidade da ventilação natural nas habitações adjacentes.

Estudos com abordagem acerca do clima urbano (Amorim, 2017; Lima e Galvani, 2020; Piselli et al., 2018; entre outros) e da poluição sonora (Chaux-álvarez e Acevedo-buitrago, 2019; Fiedler e Zannin, 2015; Lopez e Souza, 2018; entre outros) já são bastante consolidadas na comunidade científica de forma isolada, tanto no cenário mundial, quanto no cenário nacional. Entretanto, estudos integrados entre as referidas temáticas possibilitam encontrar estratégias e recomendações mais adequadas para a promoção de cidades mais saudáveis.

Jacobi (2003) defende que a construção de uma cidade saudável deve ser interdisciplinar, envolvendo estudos de conforto ambiental e a percepção da população, tendo como objetivo a manutenção da qualidade de vida para que os moradores possam vivenciar a natureza e aumentar a capacidade de mitigação do clima. Vale destacar o trabalho de Hirashima (2014), desenvolvido na cidade de Belo Horizonte, Minas Gerais, que teve como objetivo o estudo integrado para o estudo do conforto térmico e acústico no meio urbano, com a coleta de dados acústicos e climáticos, além da aplicação de formulários relativos às percepções dos usuários. Hirashima (2014) concluiu que o aumento do desconforto acústico pode implicar no aumento do desconforto térmico, e vice-versa, detectando assim, prováveis efeitos combinados entre a percepção acústica e térmica.

O documento "Nature-Based Solutions \& Re-Naturing Cities" elaborado pela União Europeia traz soluções baseadas na natureza para promover uma urbanização sustentável e resiliente às 
mudanças climáticas nos países membros. Essas soluções buscam melhorar a saúde da população, a gestão de bacias hidrográficas e a restauração de ecossistemas com eficiência energética e de recursos, considerando a economia e a participação social (European Commission, 2015). GilesCorti et al. (2016), elencam em seu estudo sobre planejamento urbano e saúde oito intervenções para construção de uma cidade saudável que envolve mudanças no sistema público de transporte e modo de vida dos cidadãos com o incentivo de caminhada, uso de bicicleta e transporte coletivo.

A pesquisa realizada por Nieuwenhuijsen (2018) associa o planejamento urbano, de transporte e a saúde dos habitantes. Mostrando que o modo como as pessoas se locomovem afeta a exposição ambiental e o estilo de vida, os quais estão interligados à mortalidade e ao aparecimento de enfermidades como infarto e obesidade. Assim, conclui-se que o planejamento urbano e de transporte tem um efeito importante sobre a saúde cardiovascular e seus fatores de risco.

A partir do exposto, o presente artigo tem como objetivo discutir o panorama da produção científica, entre os anos de 1977 e 2020, de investigações sobre clima urbano e poluição sonora, cuja abordagem entre duas temáticas tenha ocorrido de forma integrada e complementar, com vistas a compreender como a conexão entre ambas tem sido considerada ao longo dos anos no campo do conforto ambiental.

\section{Materiais e Métodos}

Para realização desta pesquisa, foram utilizados procedimentos técnicos da pesquisa bibliográfica e pesquisa-ação. Segundo Severino (2007), na pesquisa bibliográfica é formulada uma análise a partir de documentos científicos já publicados como livros, periódicos e artigos científicos. Já na pesquisa-ação, o pesquisador interfere na investigação ao realizar um diagnóstico ou análise de determinada busca. Metodologia similar foi adotada nos trabalhos elaborados por Mota et al. (2019) e Valmorbida et al. (2014).

Objetivando apresentar um panorama da produção científica relativa à poluição sonora e ao clima urbano, foram adotadas as seguintes etapas metodológicas: (i) seleção de artigos brutos com definição de critérios de busca nas bases de dados Science Direct (Elsevier), Scopus (Elsevier), Web of Science e Scielo; (ii) filtragem de artigos brutos por meio de processo de seleção ou descarte; e (iii) análise e tabulação dos resultados por meio de gráficos e tabelas. A filtragem dos artigos selecionados foi subdividida em: (a) artigos repetidos; (b) alinhamento dos títulos com o tema e (c) aderência da metodologia ao tema.

As bases de dados foram acessadas por meio do Portal de Periódicos Capes. O levantamento de dados foi executado nos meses de novembro e dezembro de 2020. A primeira estratégia de busca foi a consideração da expressão: "noise pollution" AND climate, tendo sido encontrado o total de 417 documentos na base Scopus (Elsevier). No entanto, observou-se que seria necessário especificar a escala a ser considerada: do edifício ou urbana. Assim, a fim de refinar a busca, foi utilizada a expressão: "noise pollution" AND climate AND urban.

Com o portfólio bibliográfico em mãos, foi possível selecionar os artigos, com a finalidade de identificar o grau de correlação entre as temáticas: poluição sonora e clima urbano. Inicialmente, os artigos foram exportados para o gerenciador de referências Mendeley para exclusão dos documentos repetidos e início da filtragem. Assim, foram eliminados os artigos repetidos e foi realizada a análise da aderência, inicialmente, quanto ao título, para posteriormente, realizar a verificação de aderência quanto à metodologia, ou seja, verificar se realmente quais artigos baseiam a sua metodologia em um estudo integrado entre as duas temáticas em questão.

\section{Resultados e Discussões}

\subsection{Aspectos Gerais da Busca e Seleção}

A partir da expressão de busca: "noise pollution" AND climate AND urban, o número de documentos científicos identificados foi de: (a) Science Direct, 1481 artigos, (b) Scopus, 141 artigos 
e (c) Web of Science, 41 artigos, totalizando 1663 artigos, publicados entre os anos entre os anos de 1977 e 2020. Na Scielo não foi obtido nenhum documento. Após a eliminação dos artigos repetidos, chegou-se ao número de 1548 publicações a serem revisadas. Posteriormente, os títulos dos artigos foram lidos para verificação da aderência, restando 214 alinhados aos temas, conforme pode ser observado na Tabela 1 a seguir.

Tabela 1 - Seleção de artigos que correlacionam as temáticas: clima urbano e poluição sonora, a partir da busca "noise pollution" AND climate AND urban.

\begin{tabular}{|c|c|c|c|c|}
\hline Bases de dados & $\begin{array}{l}\text { Artigos da } \\
\text { busca }\end{array}$ & Duplicatas & $\begin{array}{c}\text { Aderência } \\
\text { do título }\end{array}$ & $\begin{array}{c}\text { Aderência da } \\
\text { metodologia }\end{array}$ \\
\hline Science Direct (Elsevier) & 1481 & \multirow{4}{*}{115} & 149 & 8 \\
\hline Scopus (Elsevier) & 141 & & 59 & 6 \\
\hline Web of Science & 41 & & 6 & 0 \\
\hline Scielo & 0 & & 0 & 0 \\
\hline TOTAL & 1663 & 1548 & 214 & 14 \\
\hline
\end{tabular}

Fonte: Elaboração própria.

Conforme mencionado, foram identificados e selecionados 14 artigos científicos que apresentaram uma abordagem integrada das duas temáticas em questão: clima urbano e poluição sonora. As publicações encontradas datam de 2007 até o ano 2020, conforme pode ser observado Quadro 1. Observa-se que todos os artigos identificados se referem a publicações em revistas/periódicos.

Quadro 1 - Seleção de artigos que correlacionam as temáticas: clima urbano e poluição sonora, a partir da busca "noise pollution" AND climate AND urban.

\begin{tabular}{|c|c|c|c|c|c|}
\hline Autor & Ano & Título & Periódico & Área & $\begin{array}{c}\text { Local do } \\
\text { Objeto de } \\
\text { Pesquisa }\end{array}$ \\
\hline $\begin{array}{l}\text { Tang, U. W. } \\
\text { Wang, Z. S. }\end{array}$ & 2007 & $\begin{array}{l}\text { Influences of urban } \\
\text { forms on traffic- } \\
\text { induced noise and } \\
\text { air pollution: } \\
\text { Results from a } \\
\text { modelling system }\end{array}$ & $\begin{array}{c}\text { Environmental } \\
\text { Modelling and } \\
\text { Software }\end{array}$ & $\begin{array}{l}\text { 1.Ciências da } \\
\text { Computação } \\
\text { 2.Ciências } \\
\text { Ambientais }\end{array}$ & $\begin{array}{c}\text { Macau, } \\
\text { Macao, } \\
\text { China }\end{array}$ \\
\hline $\begin{array}{c}\text { Su, Y.X } \\
\text { Huang, G.Q. } \\
\text { Chen, X.Z. } \\
\text { Chen, S.S. } \\
\text { Li, Z.S. }\end{array}$ & 2011 & $\begin{array}{l}\text { Research progress } \\
\text { in the eco- } \\
\text { environmental } \\
\text { effects of urban } \\
\text { green spaces }\end{array}$ & Acta Ecolo & $\begin{array}{l}\text { 1.Agricultura e } \\
\text { Ciências } \\
\text { Biológicas } \\
\text { 2.Ciências } \\
\text { Ambientais }\end{array}$ & $\begin{array}{l}\text { Artigo de } \\
\text { revisão }\end{array}$ \\
\hline $\begin{array}{l}\text { Islam, M. N. } \\
\text { Rahman, K. } \\
\text { Bahar, M. M. } \\
\text { Habib, M. A. }\end{array}$ & 2012 & $\begin{array}{c}\text { Pollution } \\
\text { attenuation by } \\
\text { roadside greenbelt }\end{array}$ & $\begin{array}{c}\text { Urban } \\
\text { Forestry and } \\
\text { Urban } \\
\text { Greening }\end{array}$ & $\begin{array}{c}\text { 1.Agricultura e } \\
\text { Ciências } \\
\text { Biológicas }\end{array}$ & $\begin{array}{l}\text { Khulna, } \\
\text { Bangladesh }\end{array}$ \\
\hline
\end{tabular}


Ando, K. Hattori, N. in and around urban areas
2.Ciências

Ambientais

\begin{tabular}{|c|c|c|c|c|c|}
\hline $\begin{array}{l}\text { Kim, K. H. } \\
\text { Ho, D. X. } \\
\text { Brown, R. J.C. } \\
\text { Oh, J. M. } \\
\text { Park, C. G. } \\
\text { Ryu, I. C. }\end{array}$ & 2012 & $\begin{array}{c}\text { Some insights into } \\
\text { the relationship } \\
\text { between urban air } \\
\text { pollution and noise } \\
\text { levels }\end{array}$ & $\begin{array}{l}\text { Science of the } \\
\text { Total } \\
\text { Environment }\end{array}$ & $\begin{array}{l}\text { 1.Ciências } \\
\text { Ambientais }\end{array}$ & $\begin{array}{c}\text { Seoul, Coréia } \\
\text { do Sul }\end{array}$ \\
\hline $\begin{array}{l}\text { Silva, L. T. } \\
\text { Mendes, J. F. } \\
\text { G. }\end{array}$ & 2012 & $\begin{array}{l}\text { City Noise-Air: An } \\
\text { environmental } \\
\text { quality index for } \\
\text { cities }\end{array}$ & $\begin{array}{c}\text { Sustainable } \\
\text { Cities and } \\
\text { Society }\end{array}$ & $\begin{array}{l}\text { 1.Energia } \\
\text { 2.Engenharia } \\
\text { 3.Ciências } \\
\text { Sociais }\end{array}$ & $\begin{array}{l}\text { Viana do } \\
\text { Castelo, } \\
\text { Portugal }\end{array}$ \\
\hline $\begin{array}{l}\text { Gratani, L. } \\
\text { Varone, L. }\end{array}$ & 2013 & $\begin{array}{c}\text { Carbon } \\
\text { sequestration and } \\
\text { noise attenuation } \\
\text { provided by hedges } \\
\text { in Rome: the } \\
\text { contribution of } \\
\text { hedge traits in } \\
\text { decreasing } \\
\text { pollution levels }\end{array}$ & $\begin{array}{c}\text { Atmospheric } \\
\text { Pollution } \\
\text { Research }\end{array}$ & $\begin{array}{l}\text { 1.Ciências da } \\
\text { Terra e } \\
\text { planetárias } \\
\text { 2.Ciências } \\
\text { Ambientais }\end{array}$ & Roma, Itália \\
\hline $\begin{array}{l}\text { Cohen, P. } \\
\text { Potchter, O. } \\
\text { Schnell, I. }\end{array}$ & 2014 & $\begin{array}{c}\text { The impact of an } \\
\text { urban park on air } \\
\text { pollution and noise } \\
\text { levels in the } \\
\text { Mediterranean city } \\
\text { of Tel-Aviv, Israel }\end{array}$ & $\begin{array}{c}\text { Environmental } \\
\text { Pollution }\end{array}$ & $\begin{array}{l}\text { 1.Ciências } \\
\text { Ambientais } \\
\text { 2.Farmacologia, } \\
\text { Toxicologia e } \\
\text { Farmacêutica }\end{array}$ & $\begin{array}{l}\text { Tel Aviv, } \\
\text { Israel }\end{array}$ \\
\hline $\begin{array}{l}\text { Weber, N. } \\
\text { Haase, D. } \\
\text { Franck, U. }\end{array}$ & 2014 & $\begin{array}{l}\text { Assessing modelled } \\
\text { outdoor traffic- } \\
\text { induced noise and } \\
\text { air pollution } \\
\text { around urban } \\
\text { structures using the } \\
\text { concept of } \\
\text { landscape metrics }\end{array}$ & $\begin{array}{l}\text { Landscape } \\
\text { and Urban } \\
\text { Planning }\end{array}$ & $\begin{array}{l}\text { 1.Ciências } \\
\text { Ambientais }\end{array}$ & $\begin{array}{l}\text { Leipzig, } \\
\text { Alemanha }\end{array}$ \\
\hline $\begin{array}{l}\text { Lera-López, F. } \\
\text { Sánchez, M. } \\
\text { Faulin, J. } \\
\text { Cacciolatti, L. }\end{array}$ & 2014 & $\begin{array}{l}\text { Rural environment } \\
\text { stakeholders and } \\
\text { policy making: } \\
\text { Willingness to pay } \\
\text { to reduce road } \\
\text { transportation } \\
\text { pollution impact in } \\
\text { the Western } \\
\text { Pyrenees }\end{array}$ & $\begin{array}{c}\text { Transportation } \\
\text { Research Part } \\
\text { D: Transport } \\
\text { and } \\
\text { Environment }\end{array}$ & $\begin{array}{l}\text { 1.Engenharia } \\
\text { 2.Ciências } \\
\text { Ambientais } \\
\text { 3.Ciências } \\
\text { Sociais }\end{array}$ & $\begin{array}{l}\text { Região de } \\
\text { Pirineus, } \\
\text { Espanha }\end{array}$ \\
\hline
\end{tabular}




\begin{tabular}{|c|c|c|c|c|c|}
\hline $\begin{array}{c}\text { Echevarria } \\
\text { Sanchez, G.M. } \\
\text { Van } \\
\text { Renterghem, } \\
\text { T. } \\
\text { Thomas, P. } \\
\text { Botteldooren, } \\
\text { D. }\end{array}$ & 2016 & $\begin{array}{l}\text { The effect of street } \\
\text { canyon design on } \\
\text { traffic noise } \\
\text { exposure along } \\
\text { roads }\end{array}$ & $\begin{array}{l}\text { Building and } \\
\text { Environment }\end{array}$ & $\begin{array}{l}\text { 1.Engenharia } \\
\text { 2.Ciências } \\
\text { Ambientais } \\
\text { 3.Ciências } \\
\text { Sociais }\end{array}$ & $\begin{array}{l}\text { Ghent, } \\
\text { Bélgica }\end{array}$ \\
\hline $\begin{array}{l}\text { De Carvalho, } \\
\text { R.M. } \\
\text { Szlafsztein, } \\
\text { C.F. }\end{array}$ & 2019 & $\begin{array}{c}\text { Urban vegetation } \\
\text { loss and ecosystem } \\
\text { services: The } \\
\text { influence on } \\
\text { climate regulation } \\
\text { and noise and air } \\
\text { pollution }\end{array}$ & $\begin{array}{l}\text { Environmental } \\
\text { Pollution }\end{array}$ & $\begin{array}{l}\text { 1.Ciências } \\
\text { Ambientais } \\
\text { 2.Farmacologia, } \\
\text { Toxicologia e } \\
\text { Farmacêutica }\end{array}$ & Belém, Brasil \\
\hline $\begin{array}{c}\text { Kousis, I. } \\
\text { Pisello, A.L. }\end{array}$ & 2020 & $\begin{array}{l}\text { For the mitigation } \\
\text { of urban heat } \\
\text { island and urban } \\
\text { noise island: Two } \\
\text { simultaneous sides } \\
\text { of urban discomfort }\end{array}$ & $\begin{array}{l}\text { Environmental } \\
\text { Research } \\
\text { Letters }\end{array}$ & $\begin{array}{l}\text { 1.Energia } \\
\text { 2.Ciências } \\
\text { Ambientais } \\
\text { 3.Medicina }\end{array}$ & $\begin{array}{l}\text { Artigo de } \\
\text { revisão }\end{array}$ \\
\hline $\begin{array}{l}\text { Soydan, O., } \\
\text { Benliay, A. }\end{array}$ & 2020 & $\begin{array}{l}\text { Noise pollutions } \\
\text { depending on } \\
\text { weather conditions } \\
\text { in Antalya city }\end{array}$ & $\begin{array}{c}\text { Fresenius } \\
\text { Environmental } \\
\text { Bulletin }\end{array}$ & $\begin{array}{l}\text { 1.Ciências } \\
\text { Ambientais }\end{array}$ & $\begin{array}{l}\text { Antalya, } \\
\text { Turquia }\end{array}$ \\
\hline $\begin{array}{c}\text { Baquero } \\
\text { Larriva, M.T., } \\
\text { Higueras, E. }\end{array}$ & 2020 & $\begin{array}{c}\text { Health risk for } \\
\text { older adults in } \\
\text { Madrid, by outdoor } \\
\text { thermal and } \\
\text { acoustic comfort }\end{array}$ & Urban Climate & $\begin{array}{c}\text { 1.Ciências da } \\
\text { terra e } \\
\text { planetárias } \\
\text { 2.Ciências } \\
\text { ambientais } \\
\text { 3.Ciências } \\
\text { Sociais }\end{array}$ & $\begin{array}{l}\text { Madrid, } \\
\text { Espanha }\end{array}$ \\
\hline
\end{tabular}

Fonte: Elaboração própria.

Após o refinamento dos dados pelas palavras-chaves e agrupamento da produção científica por áreas comuns, foi possível observar que a maior parte das referências encontradas estão inseridas na área de Ciências Ambientais, sendo apontada como área de estudo em 13 artigos, dos 14 documentos considerados na presente pesquisa, seguido da área de Ciências Sociais, presente em 4 artigos e Engenharia, presente em 3 artigos. Lembrando que a classificação de áreas de estudo foi fornecida pelas bases de dados e que, os artigos em estudo tiveram uma, duas ou três áreas enquadradas.

Quanto ao local dos objetos de estudo dos autores dos artigos selecionados, há dois artigos desenvolvidos na Espanha, com os trabalhos de Lera-López et al. (2014) e Baquero e Higueras (2020), enquanto os demais artigos, sendo um artigo por país, foram aplicados a cidades na China (Tang e Wang, 2007), Bangladesh (Islam et al., 2012), Coréia do Sul (Kim et al., 2012), Portugal 
(Silva e Mendes, 2012), Itália (Gratani e Varone, 2013), Israel (Cohen et al., 2014), Alemanha (Weber et al., 2014), Bélgica (Echevarria Sánchez et al., 2016), Turquia (Soydan e Benliay, 2020) e Brasil (De Carvalho e Szlafsztein, 2019) . Além disso, há dois artigos de revisão, artigos de Su et al. (2011) e Kousis e Pisello (2020).

A partir das palavras-chaves fornecidas pelos bancos de dados, foi possível elaborar a nuvem de palavras (Figura 1), na qual pode-se observar as palavras mais recorrentes nos artigos analisados. A palavra-chave mais presente foi poluição do ar, seguido de poluição atmosférica e ruído.

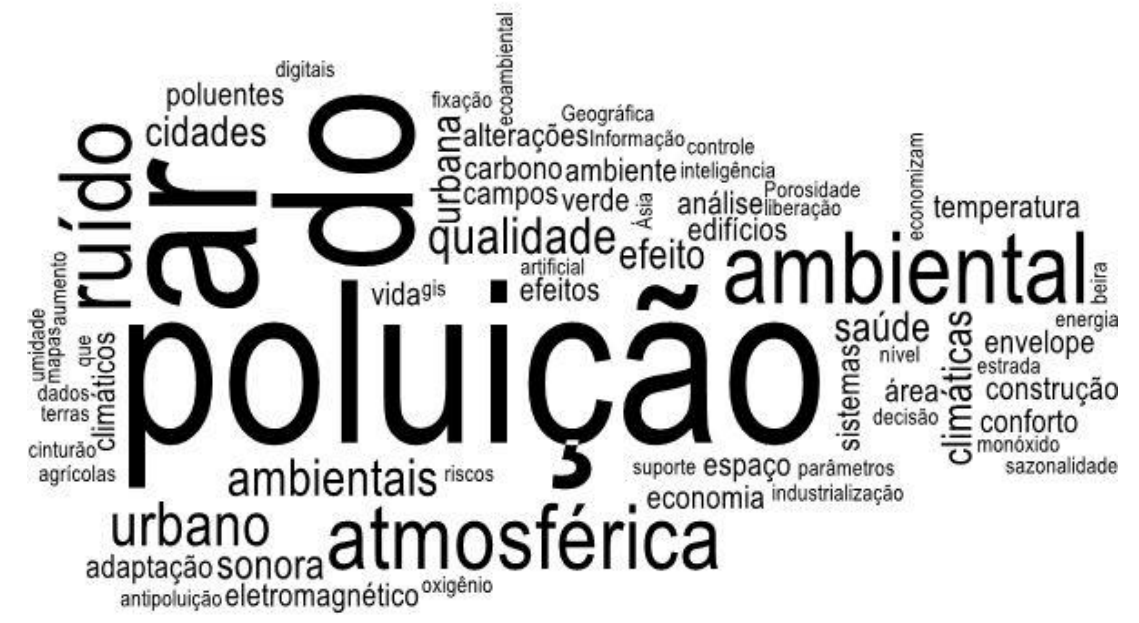

Figura 1 - Nuvem de palavras-chave, a partir dos artigos selecionados para estudo

\subsection{Correlações entre as Temáticas: Clima Urbano e Poluição Sonora}

Dentre os artigos científicos consultados, a pesquisa de Tang e Wang (2007) investigou como quatro formas urbanas existentes na Península de Macau, na China, influenciam na poluição atmosférica e sonora, com o ruído de tráfego como principal fonte. Por meio de um protótipo de sistema de modelagem, que integra o modelo de ruído do tráfego rodoviário, a poluição do ar operacional, mapas digitais da rede viária, layout de construção e informações topográficas e um modelo de paisagem urbana, investigou-se a qualidade do ar e níveis de ruído em cada morfologia urbana. Os resultados mostraram que nas áreas onde as vias eram mais estreitas, o tráfego era limitado, diminuindo os níveis de ruído, porém o cânion urbano mais profundo obteve maiores concentrações de monóxido de carbono (CO).

$\mathrm{Su}$ et al. (2011) realizaram um levantamento de trabalhos similares que confirmam a importância da vegetação demonstrando que os espaços verdes urbanos podem absorver dióxido de carbono, liberarem oxigênio, baixar a temperatura do ar, aumentar a umidade do ar, reduzir a concentração de poeira e o nível de ruído, enquanto podiam proteger a biodiversidade e reduzir a poluição do ar através da fotossíntese, absorção, função tampão, transpiração e evapotranspiração da vegetação. Com base nas diferenças dos métodos e conteúdos de pesquisa existentes, o estudo resumiu e avaliou sistematicamente as pesquisas sobre os efeitos eco-ambientais de espaços verdes urbanos. As dificuldades, deficiências e direções-chave do estudo atual foram indicadas para referências a futuras pesquisas aliadas.

Em 2012, três artigos atenderam totalmente à busca, o primeiro estudo analisou como os cinturões verdes podem ser ferramentas eficazes para mitigar a poluição sonora e do ar induzida pelo tráfego (Islam et al., 2012). Considerou-se o estado da vegetação, a densidade sazonal da copa, o cânion urbano, o ruído de tráfego e a porcentagem de remoção de partículas suspensas totais (PST) de duas vias semelhantes fisicamente, uma em Khulna, Bangladesh, e outra fora da cidade. Os resultados mostraram que os cinturões verdes contribuíram muito para reduzir a poluição do PST e chegou a $65 \%$. A redução do nível de ruído também foi alcançada em até $17 \mathrm{~dB}$ quando comparada à área aberta. 
A fim de avaliar a relação entre ruído e poluição do ar, as concentrações dos poluentes atmosféricos mais críticos também foram medidas nas estações de monitoramento da qualidade do ar (MQA) próximas a cada local de monitoramento de ruído (Kim et al., 2012). Os resultados confirmaram a relação entre os dois tipos de poluição, os quais mostram diferenças significativas entre os níveis de ruído e poluentes atmosféricos em áreas rodoviárias, superiores às não rodoviárias.

Silva e Mendes (2012) propõem um modelo de longo prazo para avaliação combinada da poluição atmosférica e sonora urbana com base em valores limites de nível de ruído e concentração de poluentes atmosféricos recomendados pela OMS, chamado City Noise-Air. O estudo foi realizado em Viana do Castelo, em Portugal, onde foram observados cinco principais poluentes do ar e dois cenários sonoros, um no verão, outro no inverno. O modelo gerou um índice resumido da qualidade do ar, o qual é de fácil compreensão para o público em geral, com valores em porcentagens nos bairros que ultrapassaram os limites.

Em Roma, desenvolveu-se um estudo sobre quatro espécies de cercas vivas utilizadas amplamente na infraestrutura verde da cidade para a redução da concentração de $\mathrm{CO} 2$ e o nível de ruído em cada um dos locais. A capacidade mensal de sequestro de $\mathrm{CO} 2$ foi calculada multiplicando a fotossíntese total por cerca viva pelo tempo total de atividade fotossintética (em horas) por mês. A análise de regressão múltipla previu a atenuação do ruído por uma combinação linear da área foliar total, densidade foliar total e área de massa foliar dos tipos de cobertura vegetal considerados. Os resultados evidenciaram quais espécies foram mais eficientes para mitigar os efeitos da poluição (Gratani e Varone, 2013).

Também relacionado à vegetação, a pesquisa de Cohen et al. (2014) avaliou a influência dos parques urbanos na qualidade do ar e no ruído na cidade de Tel-Aviv, em Israel, por meio da investigação de um parque urbano, uma praça urbana e um cânion urbano. Houve monitoramento simultâneo de vários poluentes atmosféricos e medição de níveis de ruído. Pode-se observar que o benefício do parque urbano na redução das concentrações de NOx (óxido de azoto) e PM10 (partículas respiratórias) é mais significativo do que a desvantagem de níveis elevados de O3 (ozônio). Além disso, os parques urbanos podem reduzir o ruído em até $5 \mathrm{~dB}(\mathrm{~A})$.

Através do conceito de métricas da paisagem, algoritmos que quantificam características espaciais específicas de elementos urbanos usando mapas temáticos, foi possível explicar o nível do ruído externo e a concentração de partículas de poluição do ar no centro da Alemanha. Relacionouse esses elementos com o tipo de uso e ocupação do solo, observando que os maiores índices estavam em áreas residenciais altamente verticalizadas ou em casas antigas geminadas. Logo, concluiu-se que as métricas da paisagem são muito úteis na previsão de ruído e exposição de partículas poluentes, auxiliando no planejamento urbano (Weber et al., 2014).

No estudo de Lera-López et al. (2014), além das medições dos níveis de ruído e poluição do ar, também houve aplicação de questionários em 900 residentes de 14 cidades rurais próximas de rodovias para conhecer a disposição de pagar dos cidadãos para reduzir o ruído e a poluição do ar. A região de estudo foi Pirenéus, que forma uma fronteira natural entre a Espanha e o resto da Europa, mais de 150.000 veículos cruzam diariamente esta área. Os resultados indicam que as partes interessadas que vivem perto de estradas principais têm incentivos mais elevados para compensar os custos ambientais. Além disso, cidadãos mais jovens, mais educados e mais conscientes do meio ambiente estão dispostos a pagar mais para reduzir as externalidades,

Já no estudo de Echevarria Sánchez et al. (2016), os autores abordam o efeito do projeto do cânion de rua na exposição ao ruído do tráfego ao longo das estradas, defendendo que arquitetos e urbanistas tomam decisões sobre regulamentos urbanos que definem a morfologia das ruas e edifícios sem levar este aspecto em consideração. Os receptores, tanto ao longo das fachadas, quanto nas calçadas, foram considerados em 42 casos. Os resultados numéricos demonstram que a forma do edifício, a geometria da rua e a presença de mobiliário urbano podem ter um forte impacto sobre a exposição ao ruído das pessoas. Tendo como resultado a comprovação do que o projeto cuidadoso das fachadas dos edifícios e da geometria das ruas poderia melhorar o clima sonoro para as pessoas que vivem e andam ao longo das ruas urbanas movimentadas e deveria ser considerado no projeto de futuras ruas urbanas. 
Correlacionando as duas temáticas e abordando a influência das áreas verdes urbanas na regulação do clima e dos níveis de poluição sonora, vale ressaltar o trabalho de De Carvalho e Szlafsztein (2019), que buscou identificar a perda de vegetação urbana e relacioná-la com a prestação de serviços ecossistêmicos de redução da qualidade do ar, redução da poluição do ar e regulação do clima. Este trabalho teve como objeto de estudo a cidade de Belém, Pará, Brasil, com a coleta de dados relativos à distribuição espacial e temporal da cobertura vegetal de 1986 a 2009 na cidade. Os dados sobre poluição do ar, poluição sonora e temperatura foram terceirizados em trabalhos anteriores. Constatou-se a perda de cobertura vegetal nas áreas urbanizadas foi expressiva ao longo dos anos em estudo, verificando assim, que com o aumento da degradação de áreas naturais no ambiente urbano, a provisão de serviços ecossistêmicos diminuiu, o que consequentemente prejudicou a regulação do clima e a poluição do ar e sonora.

O trabalho de Kousis e Pisello (2020) apresenta uma abordagem simultânea das duas temáticas no contexto urbano, ou seja, do clima urbano, com o enfoque nas ilhas de calor urbanas e poluição sonora, que eles chamam de ilha de ruído urbana. Os referidos autores consideram que as ações antrópicas na cidade são capazes de interferir no bem-estar das pessoas, a exemplo do aquecimento agressivo ocasionado pelas ilhas de calor urbana, além do desconforto gerado pela poluição sonora, impactando na vida social e nas condições de saúde dos centros urbanos. Trata-se de um artigo de revisão, que busca preencher a lacuna existente no campo de estudo, que estuda, frequentemente, as temáticas em separado, ou seja, entre a análise apenas termofísica sobre a mitigação de ilhas de calor e a análise apenas acústica da poluição sonora urbana. Kousis e Pisello (2020) defendem o uso de superfícies adaptativas e multifuncionais, incluindo pavimentos urbanos e envolventes de construção, a fim de mitigar as ilhas de calor e de ruído. O propósito final dos autores é promover investigação e inovação para cidades mais habitáveis por meio de uma visão mais holística.

No estudo realizado por Soydan e Benliay (2020) foram analisados os níveis de ruído no boulevard de 100 anos na cidade de Antalya, Turquia, e através do método de Redes Neurais Artificiais foi possível determinar os valores de previsão do vento para os próximos anos. As medições de ruído foram realizadas nos três turnos, diurno (07h00-19h00), noturno (19h00-23h00) e na madrugada (23h00-07h00), as quais foram transferidas para o software de monitoramento do vento. Foram realizados doze mapas de ruído para os períodos diurno e noturno, além de tabelas de estimativa de vento e ruído, o nível máximo catalogado chegou a 82,68 $\mathrm{dB}(\mathrm{A})$, constatando a poluição sonora. Assim, a partir destes mapas, foram identificadas áreas com problemas em termos de poluição sonora e elaboradas recomendações de mitigação.

Baquero e Higueras (2020) analisaram a percepção do conforto térmico e acústico em idosos em espaços públicos na cidade de Madrid (Espanha). As análises objetivas, por meio de medição dos níveis de pressão sonora, Temperatura Equivalente Fisiológica (PET) e Índice Térmico Universal (UTCI), e subjetivas, com aplicação de 413 questionários, foram comparadas. Os resultados mostraram que apesar dos usuários considerarem o ambiente como confortável, os índices ultrapassaram os limites recomendados pela OMS, alertando ao risco significativo para a saúde com a exposição à poluição sonora e temperaturas extremas diárias.

\section{Conclusão}

A partir da amostragem de artigos científicos analisados, verificou-se a tendência de que os trabalhos mais recentes, publicados em meados dos anos 2000 até os dias atuais, há uma tentativa maior de cruzamento entre as temáticas, de maneira que, quanto mais antigo, mais os trabalhos tendem a estudar de maneira mais isolada uma das áreas. De uma maneira geral, foram observados trabalhos que correlacionam ilhas de calor e poluição sonora, mudanças climáticas e ruído urbano, percepção do conforto térmico e acústico em espaços públicos, influência das áreas verdes urbanas na regulação do clima e dos níveis de poluição sonora, entre outros.

Como proposta para trabalhos futuros, podem ser realizados trabalhos com buscas em outras bases de dados científicos, bem como com mais expressões de busca. Além disso, outros trabalhos 
podem ser desenvolvidos que estudem as smart cities, visto que é um campo de estudo amplo e crescente, que visa a análise integrada e mais holística dos parâmetros ambientais.

Apesar da expressividade de produção acadêmica desenvolvida nas áreas de estudo, em separado, evidenciou-se que poucos trabalham abordam a temática de forma integrada, o que pode se configurar como uma lacuna no âmbito do conforto ambiental urbano. Por fim, é necessário desenvolver pesquisas mais integradas, que possam considerar os inúmeros aspectos envolvidos no ambiente urbano, com a finalidade de construção de diretrizes e instrumentos projetuais e tomada de decisão no planejamento de cidades mais habitáveis e saudáveis.

\section{Referências}

Amorim, M. C. de C. T. (2017). Detecção remota de ilhas de calor superficiais: Exemplos de cidades de porte médio e pequeno do ambiente tropical, Brasil, Finisterra, LII, n. 105, p. 111-133. doi: 10.18055/Finis6888

Baquero Larriva, M.T., Higueras, E. (2020). Health risk for older adults in Madrid, by outdoor thermal and acoustic comfort. Urban Climate, v. 34. doi: 10.1016/j.uclim.2020.100724

Chaux-álvarez, L. M., Acevedo-buitrago, B. (2019) Evaluación de ruido ambiental en alrededores a centros médicos de la localidad Barrios Unidos, Bogotá. Revista Científica, 35(2), 234-246. doi: https://doi.org/10.14483/23448350.13983

Cohen, P., Potchter, O., Schnell, I. (2014). The impact of an urban park on air pollution and noise levels in the Mediterranean city of Tel-Aviv, Israel. Environmental Pollution, [S. 1.], v. 195, p. 73-83. doi: 10.1016/j.envpol.2014.08.015

De Carvalho, R. M., Szlafsztein, C. F. (2019). Urban vegetation loss and ecosystem services: The influence on climate regulation and noise and air pollution. Environmental Pollution, [S. 1.], v. 245, p. 844-852. doi: https://doi.org/10.1016/j.envpol.2018.10.114

Echevarria Sánchez, G., Renterghem, T. V., Thomas, P., Botteldooren, D. (2016). The effect of street canyon design on traffic noise exposure along roads. Building and Environment. doi: 10.1016/j.buildenv.2015.11.033. corpus id: 111020857

European Commission. (2015). Nature-Based Solutions \& Re-Naturing Cities - Final Report of the Horizon 2020 Expert Group on 'Nature-Based Solutions and Re-Naturing Cities', Office of the European Union, Luxembourg.

Fiedler, P. E. K., Zannin, P. H. T. (2015). Evaluation of noise pollution in urban traffic hubs - Noise maps and measurements, Environmental Impact Assessment Review. doi: 10.1016/j.eiar.2014.09.014

Giles-corti, B., Vernez-Moudon, A., Reis, R., Turrell, G., Dannenberg, A. L., Badland, H., Foster, S., Lowe, M., Sallis, J. F. Stevenson, M., Owen, N. (2016). City planning and population health: a global challenge. The Lancet 388 (10062), 2912-2924. doi: 10.1016/S01406736(16)30066-6

Gratani, L., Varone, L. (2013). Carbon sequestration and noise attenuation provided by hedges in Rome: the contribution of hedge traits in decreasing pollution levels. Atmospheric Pollution Research, [S. 1.], v. 4, n. 3, p. 315-322. doi: 10.5094/APR.2013.035

Guedes, I. C. M., Bertoli, S. R., Zannin, P. H. T. (2011). Influence of urban shapes on environmental noise: A case study in Aracaju - Brazil, Sci Total Environ. doi:10.1016/j.scitotenv.2011.10.018

Hirashima, S. Q. da S. (2014). Percepção sonora e térmica e avaliação de conforto em espaços urbanos abertos do município de Belo Horizonte - MG. Tese de Doutorado, Universidade de São Paulo, São Paulo, São Paulo, SP, Brasil.

Islam, M. N., Rahman, K. S., Bahar, M. M., Habib, M. A., Ando, K., Hattori, N. (2012). Pollution attenuation by roadside greenbelt in and around urban areas. Urban Forestry and Urban Greening, [S. 1.], v. 11, n. 4, p. 460-464. doi: 10.1016/j.ufug.2012.06.004

Jacobi, P. (2003). Educação ambiental, cidadania e sustentabilidade. Cadernos de Pesquisa, n. 118, março/ 2003 p. 189-205. doi: 10.1590/s0100-15742003000100008 
Kim, K. H., Ho, D. X., Brown, R. J. C., Oh, J. M., Park, C. G., Ryu, I. C. (2012). Some insights into the relationship between urban air pollution and noise levels. Science of the Total Environment, [S. 1.], v. 424, p. 271-279. doi: 10.1016/j.scitotenv.2012.02.066

Kousis, I., Pisello, A. L. (2020). For the mitigation of urban heat island and urban noise island: Two simultaneous sides of urban discomfort. Environmental Research Letters, [S. 1.], v. 15, n. 10. doi: https://doi.org/10.1088/1748-9326/abaa0d

Koutra, S., Becue, V., Ioakimidis, C. S. (2019). Searching for the 'smart' definition through its spatial approach. Energy, [S. 1.]: Elsevier Ltd. https://doi.org/10.1016/j.energy.2018.12.019

Lera-lópez, F. Sánchez, M., Faulin, J., Cacciolatti, L. (2014). Rural environment stakeholders and policy making: Willingness to pay to reduce road transportation pollution impact in the Western Pyrenees. Transportation Research Part D: Transport and Environment, [S. 1.], v. 32, p. 129-142. doi: 10.1016/j.trd.2014.07.003

Lima, T. L., Galvani, E. (2020). Avaliação da ilha de calor urbana na zona norte de São Paulo: Estudo de caso do bairro Jardim Peri, Revista Brasileira de Climatologia. doi: 10.5380/abclima.v26i0.69933

Lopez, G. A. P., Souza, L. C. L. de. (2018). Urban green spaces and the influence on vehicular traffic noise control. Ambiente Construído, Porto Alegre, v. 18, n. 4, p. 161-175. ISSN 16788621 Associação Nacional de Tecnologia do Ambiente Construído. doi: http://dx.doi.org/10.1590/s1678-86212018000400299

Mota, L. C. C., Hirashima, S. Q. da S., Azevedo, R. C. (2019). Elaboração e análise de portfólio bibliográfico sobre mapeamento sonoro urbano utilizando o PROKNOW-C. XV ENCAC - XI ELACAC. João Pessoa: ENCAC.

Nieuwenhuijsen, M. J. (2018). Influence of urban and transport planning and the city environment on cardiovascular disease. Nature Reviews Cardiology. 15 (7), 432-438. doi: 10.1038/s41569018-0003-2

Pathak, V., Tripathi, B. D., Mishra, V. K. (2008). Dynamics of traffic noise in a tropical city Varanasi and its abatement through vegetation. Environ Monit Assess, 146(1-3):67-75. doi: 10.1007/s10661-007-0060-1. Epub 2007 Dec 18. PMID: 18085417

Piselli, C., Castaldo, V. L., Pigliautile, I., Pisello, A. L., Cotana, F. (2018). Outdoor comfort conditions in urban areas: On citizens' perspective about microclimate mitigation of urban transit areas, Sustainable Cities and Society, v. 39, p. 16-36. doi: 10.1016/j.scs.2018.02.004

Prata, S. L., Lins, S. C. R., Macfranklin, S. B. A., Carvalho, T. F., Leite, R. C. V., Nazareth, S. B. M. (2019). A influência da mudança em padrões de ocupação do solo sobre as condições ambientais urbanas: O caso do poço da draga, em Fortaleza-CE. XV ENCAC - XI ELACAC. João Pessoa: ENCAC.

Romero, M. A. B., Baptista, G. M. de M., Lima, E. A. de, Werneck, D. R., Vianna, E. O., Sales, G. de L. (2019). Mudanças climáticas e ilhas de calor urbanas. 1. ed. Brasília: Universidade de Brasília, Faculdade de Arquitetura e Urbanismo.

Severino, A. J. (2007). Metodologia do trabalho científico. 23. ed. São Paulo: Cortez.

Silva, L. T., Mendes, J. F. G. (2012). City Noise-Air: An environmental quality index for cities. Sustainable Cities and Society, [S. 1.], v. 4, n. 1, p. 1-11. doi: 10.1016/j.scs.2012.03.001

Souza, I. F., Barbosa, R. V. R., Silva, I. A. G., Zacarias, V. S. (2019). A influência das áreas verdes no microclima urbano: Estudo de caso em espaços públicos de Arapiraca-AL. XV ENCAC XI ELACAC. João Pessoa: ENCAC.

Soydan, O., Benliay, A. (2020) Noise pollutions depending on weather conditions in Antalya city. Fresenius Environmental Bulletin, v. 29, n. 6, p. 4282-4301. ISSN: 10184619

Su, Y., Huang, G., Chen, X., Chen, S., Li, Z. (2011). Research progress in the eco-environmental effects of urban green spaces. Acta Ecolo. Sin. 31 (23), 7287-7300. ISSN: 10000933

Tang, U. W.; Wang, Z. S. (2007). Influences of urban forms on traffic-induced noise and air pollution: Results from a modelling system. Environmental Modelling and Software, [S. 1.], v. 22, n. 12, p. 1750-1764. doi: 10.1016/j.envsoft.2007.02.003 
United Nations. (2019). Department of Economic and Social Affairs, Population Division (2019). World Population Prospects: The 2018 Revision (ST/ESA/SER.A/420). New York: United Nations.

Valmorbida, S. M. I., Ensslin, S. R., Ensslin, L., Bortoluzzi, S. C., Chaves, L. C. (2014). Mapeamento das publicações do tema gestão pública com foco nos resultados. RIC - Revista de Informação Contábil, [s/l].

Weber, N., Haase, D., Franck, U. (2014). Assessing modelled outdoor traffic-induced noise and air pollution around urban structures using the concept of landscape metrics. Landscape and Urban Planning, [S. 1.], v. 125, p. 105-116. doi: 10.1016/j.landurbplan.2014.02.018

WHO. (2011). Burden of disease from environmental noise - Quantification of healthy life years lost in Europe, WHO Regional Office for Europe, Copenhagen.

WHO. (2018). Environmental Noise Guidelines for the European Region, WHO Regional Office for Europe, Copenhagen.

WHO. (2020). World Health Organization. Climate change and human health - Urbanization and health. [s.1.]: WHO. Disponível em: <https://www.who.int/globalchange/ecosystems/urbanization/en/>. Acesso em: 14 dez. 2020. 